\title{
Review of The Neuroscience of Emotion: A New Synthesis \\ by Ralph Adolphs and David Anderson
}

Luiz Pessoa, University of Maryland, College Park, pessoa@umd.edu

During the past 150 years, the field of neuroscience has tried to uncover the neural underpinnings of a broad spectrum of mental phenomena, ranging from perception, action, cognition, motivation, and emotion. In The Neuroscience of Emotion (2018), Ralph Adolphs and David Anderson (henceforth, A\&A) address the last of these phenomena, providing an excellent, accessible survey of the current landscape of the field. In Part I of the book, A\&A tackle foundational issues and attempt to provide an overarching conceptual framework for investigating emotions based, in part, on key properties of emotion - as separate, for example, from cognition. In Part II, A\&A delve into the neurobiology of emotion in animals and humans. The section starts with an excellent chapter on the "logic of neuroscientific explanations". Part II contains the bulk of the chapters and delves into key issues, such as innate versus learning emotional processing, the link between consciousness and emotions - can an emotion be nonconscious? - among many others. Part III concludes with a conceptual discussion of the open questions in the field, as well as future directions.

\section{Wider context of the field}

Emotion researchers come in several flavors, but at first pass we can subdivide them into those focusing on human research and those on animal research. Unfortunately, this creates a chasm in the literature amounting to different implicit frameworks to study emotion. The Neuroscience of Emotion accomplishes something quite important in bringing the two together within a common framework. With each of the two authors being a major figure in their respective fields of research - Adolphs: human; Anderson: non-human - they bring to the table a unique and powerful combination.

A\&A's perspective is highly neurobiological. Importantly, they situate the research reviewed in the book within their framework for investigating emotions. To motivate their ideas, they ask a simple question: What kinds of questions do we want to ask in a neurobiology of emotion? To which they answer with the following working hypothesis: "Emotions are internal brain states that cause observable external changes in behavior; observable internal physiological changes in the state of the body; changes in other mental states; and, under some conditions and in some species, changes in what we are consciously aware of ('feelings', which, in humans, can often be verbally described)" (p. 30). 


\section{Should neuroscience explain emotion or complex behaviors?}

The Neuroscience of Emotion is an important book that weaves together key conceptual issues with findings from the human and animal literatures. In so doing, it provides an excellent, upto-date account of emotion neuroscience.

Here, I will comment on one of the book's weaknesses, as I see it: carving out emotion as a mental category and, with it, its associated neural substrate. Naturally, there is value in developing a "scientifically useful theory of emotions" (Adolphs and Andler, 2018). A researcher may be interested in studying a structure such as the amygdala or the periaqueductal gray in a set of well-defined and controlled tasks, such as during aversive classical conditioning. We can perhaps say that, under such circumstances, the researcher is studying "emotion."

But definitions, and deciding what is the proper status of emotion, are not a requirement for scientific progress. In fact, they can hinder progress if the conceptual boundaries prove to be stifling. For example, had physicists decided that it was imperative to establish if light functions as a particle or a wave, it would have been almost certainly detrimental to progress. For one, the wave-particle duality is at the core of the foundation of quantum mechanics. Consider another example from physics. According to the so-called Standard Model of particle physics, three forces explain phenomena at the scale of "small objects." The model does not include gravitation, which obviously matters for large objects. Whereas particle physics and gravitation could be considered separate areas of research, the goal to integrate all four fundamental forces in more complete models is an extremely important research goal. In this pursuit, physicists are not shunned because particle physics (for the sake of comparison, consider emotion) is different or separate from gravitation (consider cognition).

What is the aim of scientific research? In the context of The Neuroscience of Emotion, a central goal is to generate a scientifically useful theory of emotions. However, a broader goal, and one that I suggest is more important, is to develop a science "to explain the complex behaviors of people and animals" (Adolphs and Andler, 2018). And complex behaviors do not obey the boundaries of research areas - such as perception, cognition, action, emotion, and motivation - in useful ways.

Consider one of the main conclusions of the book (p. 308): "Emotions are implemented by neural mechanisms that we can discover and manipulate with neuroscience methods." Neuroscientists no doubt will agree. The key question is, of course, which neural mechanisms? The book's answer is, by and large, specific neural mechanisms centered upon key brain regions. But it is difficult to reconcile this stance with what we know about neuroanatomy. This is not to deny that some brain regions historically linked to emotion, such as the hypothalamus, amygdala, and periaqueductal gray, are indeed involved in emotion-related processing. But if we consider the massive combinatorial anatomical connectivity present in the brain (Pessoa, 2017a; Pessoa et al., 2019), particular areas can only be understood as parts of multiple circuits they participate in. Whereas some circuits are relatively small scale (say, intra-amygdala 
circuits), many are medium-to-large scale circuits. For example, the basolateral amygdala is extensively interconnected with most of the cortex, in addition to several subcortical areas; many of the pathways are bidirectional (Amaral et al., 1992). Accordingly, to cleave the amygdala from these other regions provides an extremely limited view of its function. A\&A seem to acknowledge this possibility, albeit with considerable hesitation: "It is possible that different properties, or subsets of properties, of an emotion state ... might be implemented by distinct neural components, almost certainly including structures outside the amygdala." (p. 171)

To develop the points above further, let's consider behavior now, and what would ostensibly constitute emotional behavior. During fear extinction, a conditioned stimulus (CS) no longer predicts the unconditioned one, and the animal learns this new state of the world. This leads to the extinction of the conditioned response previously triggered by the CS. The medial prefrontal cortex (PFC) plays an important role in regulating the amygdala during fear extinction (Morgan, Romanski, \& LeDoux, 1993). At first glance, fear extinction appears to fit the scheme of separate entities interacting during behavior: cognition (tied to the medial PFC) controlling emotion (tied to the amygdala) in a top-down fashion. Yet, considering the medial PFC as "top" and the amygdala as "down" does not account for the existing neuronal interactions. Multiple cell groups in the amygdala actually project to the medial MPFC whose outputs in turn influence amygdala signals. Some studies even have suggested a "top" role for amygdala neurons in the extinction process. The medial PFC is also the target from the hippocampus, an input that potentiates medial PFC signals during extinction. Furthermore, the medial PFC receives substantial inputs from the thalamus, itself a major subcortical-cortical connectivity hub. More generally, during fear extinction, signals from the amygdala, medial PFC, and several additional brain areas (including the orbitofrontal cortex) collectively determine whether or not to produce a response (for references regarding this paragraph, see Pessoa, 2019). These multi-region interactions afford greater behavioral malleability when responding to threat.

Consider a relatively standard approach of describing the multiple contributions to extinction mentioned above (Figure 1A). We could label each brain region involved in the following manner: amygdala: valence; medial PFC: regulation; hippocampus: context; thalamus: sensory and visceral signals; orbitofrontal cortex: reward and motivation. Extinction would result from the multiple inputs to the amygdala. Whereas this is a first step in acknowledging the multi-region contributions that contribute to extinction learning, I suggest that it is not sufficient. 
The diagram in Figure 1B tries to convey the idea that the brain regions collectively determine the extinction process. This type of description provides a different springboard to reasoning about the mapping between function and structure in the brain how brain regions bring about behaviors. Conceptually, part of the shift is due to the fact that the putative underlying processes (valence, regulation, context, etc.) are not separable, so they don't encode stable variables (such as "valence") that are simply pushed up or down by the other factors. Put another way, these variables are so intertwined that they are jointly determined: to understand the system, we need to consider the integration of the signals.

The scheme diagrammed in Figure 1B

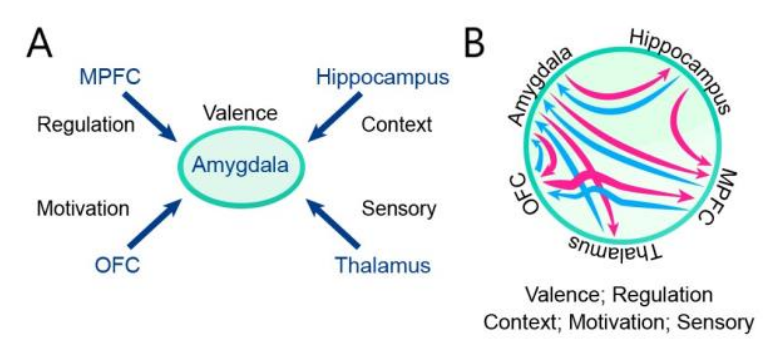

Figure 1. Contrasting explanations of fear extinction. (A) Thinking of fear extinction in terms of the topdown regulation of the amygdala by the medial prefrontal cortex, with additional variables influencing the process. (B) Schematic representation of the anatomical connections between some of the brain regions involved, emphasizing a non-hierarchical view of the processes leading to fear extinction. The descriptors "valence," "regulation," and so on, are not tied to brain areas in any straightforward one-to-one fashion. Abbreviations: MPFC, medial prefrontal cortex; OFC, orbitofrontal cortex. From Pessoa (2019) with permission. doesn't mean that all regions contribute in the same way to the behavior in question. At first glance, it appears that a lot is missed by diagramming things this way; the description appears too vague. But simplification for the sake of simplification won't help us out. Let's go back to Figure 1A, which says that the behavior in question (exhibiting fear or not upon encounter of the original CS) is a function of activity in the amygdala, itself influenced by inputs from the other areas. Figure 1B, instead, tells another story: behavior is a function of all the regions when taken together. And their bidirectional interactions imply that the flow of information is far from straightforward (such as in a standard account that the amygdala is inhibited by the medial PFC).

The boxes-and-arrows arrangement (Figure 1A; Pessoa, 2017b) also invites the interpretation that the mechanisms in question are somewhat static. Indeed, in the laboratory, one generally investigates behavior at a specific point in time, or perhaps during a narrow temporal window. But natural behaviors are dynamic, unfolding as the animal interacts with its environment. Whereas in the lab an animal may be placed in a chamber where it received shocks in the past, in nature the time frame of experiencing negative (or positive) scenarios is more gradual (unless the animal is surprised by a very sudden attack). Accordingly, a dynamic description of the underlying neural processes is not only beneficial, but necessary in general.

In summary, The Neuroscience of Emotion is an impressive volume that captures much of the excitement in this vibrant field of research. The authors expertly weave human and non- 
human research into a coherent framework, not a small accomplishment for a field that is largely fractured along this divide. While there is a lot to like about the book, I believe it would have benefited from a perspective that one of the ultimate goals of neuroscience is to explain how brain matter gives rise to complex behaviors - not just emotion.

\section{References}

Adolphs, R., \& Anderson, D. J. (2018). The neuroscience of emotion: a new synthesis. Princeton University Press.

Adolphs, R., \& Andler, D. (2018). Investigating emotions as functional states distinct from feelings. Emotion Review, 10(3), 191-201.

Amaral , D. G. , Price , J. L. , Pitkanen , A. , and Carmichael , S. T. ( 1992 ). Anatomical organization of the primate amygdaloid complex . In J. Aggleton, ed., The Amygdala: Neurobiological Aspects of Emotion, Memory, and Mental Dysfunction , 1-66. New York : WileyLiss .

Morgan, M. A., Romanski, L. M., \& LeDoux, J. E. (1993). Extinction of emotional learning: contribution of medial prefrontal cortex. Neuroscience Letters, 163(1), 109-113.

Pessoa, L. (2017a). A network model of the emotional brain. Trends in Cognitive Sciences, 21(5), 357-371.

Pessoa, L. (2017b). Cognitive-motivational interactions: Beyond boxes-and-arrows models of the mind-brain. Motivation Science, 3(3), 287.

Pessoa, L. (2019). Embracing integration and complexity: placing emotion within a science of brain and behaviour. Cognition and Emotion, 33(1), 55-60.

Pessoa, L., Medina, L., Hof, P. R., \& Desfilis, E. (2019). Neural architecture of the vertebrate brain: Implications for the interaction between emotion and cognition. Neuroscience $\mathcal{E}$ Biobehavioral Reviews, 107, 296-312. 